\title{
Automatic segmentation scheme for effective synchronization of EMG-EEG quantification
}

\author{
Suprijanto $^{(*)}$, Azizah S. Noor, Ayu G. Risangtuni, Hesty Susanti \\ Instrumentation and Control Research Group, Faculty of Industrial Technology, \\ Institut Teknologi Bandung, Bandung, West Java - Indonesia \\ ${ }^{(*)}$ supri@tf.itb.ac.id
}

Received: July 7, 2020. Revised: August 31, 2020. Accepted: September 10, 2020. Published: September 24, 2020.

\begin{abstract}
Effective segmentation of electromyography (EMG) burst that synchronizes with electroencephalography (EEG) for long-duration recording is important steps to better understand the quantification of brain-muscle connectivity in periodic motoric activities. The work proposes an alternative automatic EMG segmentation scheme consists of four main steps, i.e. denoising of EMG burst signal using discrete wavelet transform, enveloping signal using timewindows averaging of RMS amplitude, an adaptive threshold to detect start/end burst envelope with accommodation of muscle contraction characteristic and the final step is conversion enveloping signal to binary segmentation signal.

The proposed scheme is evaluated to detect contraction period/duration of EMG for the subject under repetitive holding and releasing grasp using a physiotherapy device. During exercise, the bio-amplifier board is customized to acquire simultaneous EEG and EMG from the region of flexor digitorum superficialis (FDS) of muscle and cortical motor of the brain, with total 284 EMG burst that counting by manual segmentation. The automatic segmentation can detect the total EMG burst by $6.25 \%$ error of false burst detection.

The usefulness of proposed scheme is also tested to association analysis according to the power of EMG burst and the power of mu-wave of EEG recorded on the motor cortex. The changing trend of the power of mu-wave associated with muscle relaxation, muscle contraction strength and the synchronization level on the motor cortex during exercise are analyzed with integrated information that is relevant with biofeedback concept. The results demonstrate that proposed scheme has potential to be an effective method for the evaluation of biofeedback rehabilitation exercise.
\end{abstract}

Keywords--- EMG segmentation, enveloping signal, EMGEEG quantification

\section{INTRODUCTION}

repetitive movement exercise is suggested for patients A with motor function deficits in order to help patients regain muscle function, muscle elasticity, joint range of motion and prevent muscle shortening [1]. The method to provide physiological information for patients during physical rehabilitation is called biofeedback. The majority of biofeedback research has focused on the effects of biofeedback therapy in the exercise of the upper and lower limb motor deficits in patients. For bio-feedback, muscle activities could be evaluated using electromyography (EMG). The conventional EMG quantification is focused for fatigue evaluation for specific activities [2,3] and characteristic of muscle contraction $[4,5]$.

Biofeedback quantification using electroencephalography (EEG) is also an intensive research $[6,7,8]$ related to motor activities. A $m u$-wave has reported as one of the parameters for quantifying the level of desynchronization of neuron on the motor cortex $[8,9,24]$.

Segmentation of EEG signal with synchronizing muscle activities during contraction and relaxation is important to better understand the quantification of brain-muscle connectivity in periodic motoric activities $[10,11]$.

Several approaches have proposed to find the localization and start/end (timing) of those bursts in the EMG signal, particularly for repetitive exercise and long duration recording. In [12], the algorithm to automatically detect and segment the muscle contractions existed in EMG signal was proposed based on thresholding techniques of instantaneous energy that is estimated from the time-frequency signal analysis. In this approach, the fixed threshold value only worked well for a limited condition.

Another approach of EMG segmentation is proposed in [15]. The higher-order low-pass FIR filter was applied to determine burst signal envelope. The peak detection of the signal envelope was used to estimate timing and time interval of the burst. In this work, a video recording was also used to estimate interval time of the burst using the change of marker position on the legs. Under the real situation, this approach becomes inefficient. 
The effort to increase the accuracy of the interval time of the burst was also reported in [2]. The time and frequency algorithm were applied to predict the beginning of EMG amplitude changes from relaxation to contraction. For EMG segmentation, a threshold value on the specific windows was determined by taking into account a variation of the amplitude signal. However, in real condition, those approaches are still challenging to determine the specific interval of the burst, particularly for cyclic burst during repetitive exercise.

The effort to develop an algorithm for accurately detecting interval time of the bursts using an iterative approach was proposed in [16]. An iterative algorithm was developed to select the change-points between two segments of the bursts signal based on some local statistics. This approach also attempted to estimate a local shape of EMG envelope in each bursts cycle using the likelihood-ratio. Without effective denoising EMG signal, the computation load of this approach becomes a problem, particularly in case of EMG segmentation on repetitive exercise and long duration recording.

The work proposes an alternative scheme for the automatic segmentation of an EMG burst that synchronizes with an EEG recording for periodic motoric activities. An automatic EMG segmentation scheme consists of four main steps. The first step is noise reduction processing of EMG burst signal using discrete wavelet transform (DWT). The second step is enveloping signal using time-windows averaging of RMS amplitude. The third step is converting the signal envelop to binary segmentation signal using an adaptive threshold to detect start and end of EMG burst envelope. The method to determine threshold is by accommodating muscle contraction characteristic as function of cycle repetitive contraction. The final step is converting the resulting signal envelope to a binary segmentation signal.

The proposed scheme is tested to detect the start/end contraction of burst EMG for the subject under repetitive holding and releasing grasp using a physiotherapy device. Furthermore, the usefulness of proposed scheme is also tested to analyze the relationship between the powers of EMG burst with the power of $m u$-wave as a function of periodic motoric muscle activities.

The paper is organized as follows. Section 2 briefly reviews the literatures related to some approaches for EMG segmentation and the concept behind the proposed scheme presented. In section 3, the methodology of the proposed scheme further explained and followed by the explanation of the experiment design. Finally, the experiment results and conclusion are presented in section 4.

\section{METHODS}

\section{A. EMG and EEG signal characteristic on motoric activities}

EMG signal represents the combination of action potentials from all muscle fibres of a single motor unit called motor unit action potential (MUAP). A non-invasive technique is applied by placing surface electrodes on the skin surface with respect to the motor points in the muscle.
For surface EMG signals, the amplitude is in a range between $\pm 10 \mathrm{mV}$. The higher amplitude often occurs on the period of maximum contraction, with a signal pattern which is often called EMG "burst" [18]. EMG is one of biofeedback modalities that investigates actively in medical rehabilitation exercise [12,3,5]. In exercise, repetitive/periodic movement can increase the improvement of motor skills and the smoothness of movement progressively. Information extraction from EMG burst can be used to predict the dynamic of muscle strength [13] and the process of muscle toward fatigue [14].

In contrast with EMG, the electrical activity of the brain can be recorded using EEG signal by placing gold electrodes on the scalp. This signal manifests the cortical electrical activity, measured in the order of micro-volts with restricted frequency range from 3 to $40 \mathrm{~Hz}$. Some muscle activities, for instance, the repetitive flexion and extension, are usually related to the increase of brain activity in motor coordination, attention, cognition process, and decision-making process $[8,9]$. The previous study reported that repetitive movements would trigger a de-synchronization of neuron activity in the motor cortex that called a $m u$-brain wave. The $m u$-wave is EEG signal with frequency range lies in 8 to $13 \mathrm{~Hz}$. The previous researcher has reported that $[6,7,8]$ the decrease in $m u$-wave activity over the motor cortex of the brain shows the presence of information processing related to planning, decisionmaking, and preparation of motoric activities.

Previously [10,11], coupling analysis between muscle activities and the motor cortex has been researched based on EMG and EEG data. The objective of these investigations is to better understand the brain-muscle connectivity in relation to motor function, particularly on repetitive movement. To support this research, processing scheme with the capability for comprehensive of EEG and EMG synchronization and less operator intervention is required. Next section describes the overview concepts of our proposed approach.

\section{B. Proposed Scheme}

Actually, segmentation is an effort for separating EMG signal in specific duration corresponding to the relaxation and EMG burst signal. Due to noise, determining the timing of periodic bursts signal is challenging.

In the scheme, denoising process of a raw EMG signal is applied based on multi-resolution discrete wavelet transform (DWT). Then, segmentation is processed on denoising EMG. The final output of a segmentation process is binary segmentation that is used to segment EEG signals that synchronize with the condition of muscle contraction and relaxation. The summary of processing scheme, with starting process of simultaneous EMG-EEG recording, steps for segmentation process and coupling analysis of EMG-EEG on the contraction phase and EEG on the relaxation phase is shown in Fig. 1. 


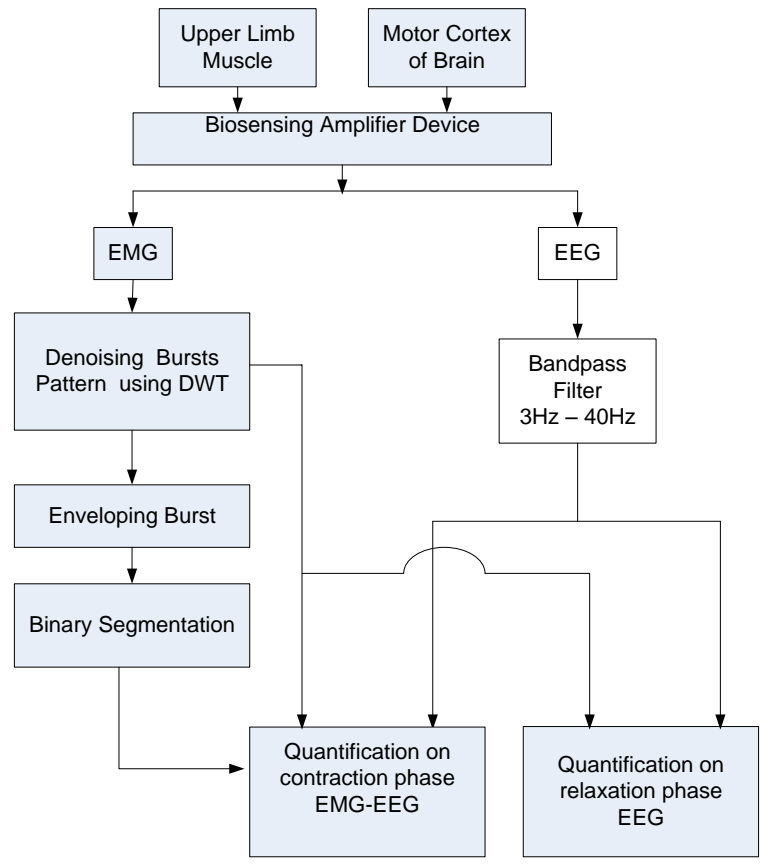

Fig. 1 The summary of processing scheme of automatic segmentation, dedicating to biofeedback coupling analysis of EMGEEG

\section{EMG Burst Denoising using DWT}

The actual EMG signal originating from muscle activities can degrade due to the mixing noise or artifacts $[13,17,18]$. These noises could arise from several factors, such as motion artifact due to muscle and electrodes movement concerning one another and the power line interference. Furthermore, anatomical factors, such as the number of muscle fibers per unit, depth and location of active fibers, and tissue thickness, could affect EMG amplitude and the lowering signal to noise ratio (SNR). For supporting automatic segmentation, noise reduction of the EMG signal is required in the pre-processing stage. The ideal objective of denoising is to optimize the reduction process of noise in a raw EMG signal and also to preserve the useful signal frequency spectrum as much as possible.

The use of a digital filter approach is a standard method for noise reduction. However, due to EMG signals non-stationary characteristics, applying various digital filter approaches is not the most efficient $[14,13,19]$.

A potential substitution for a digital filter is the discrete wavelet transform (DWT), considering the capability of studying the variable resolution both in the time and frequency domain simultaneously $[13,20]$. The multi-resolution level and basis wavelet function could be tuned more flexibly to remove noise and artifact on a raw EMG signal.

Suppose, $\mathrm{S}(\mathrm{n})$ is the raw EMG signal, the decomposition procedure on DWT is started by passing a signal through the low pass filter (LPF) $\mathbf{g}_{\mathbf{o}}$ and high pass filter (HPF) $\mathbf{h}_{\mathbf{0}}$. The LPF and HPF result in an approximation signal and a detail signal, respectively. The approximations are low-frequency components of the time series $\left(\mathrm{A}_{\mathrm{L}}(\mathrm{n})\right)$, and the details are high-frequency components $\left(D_{L}(n)\right)$, where $L$ represents the decomposition level [12]. For DWT process, the S(n) can be denoted by the approximation of $\mathrm{A}_{0}(\mathrm{n})$ at $\mathrm{L}=0$, then, the $A \mathrm{~L}$ and $D \mathrm{~L}$ of the DWT at level L can be formulated as

$A_{L}(n)=\sum_{j \in \infty} g_{o}(j-2 n) A_{L-1}(j)$

(1)

$D_{L}(n)=\sum_{j \in \infty} h_{o}(j-2 n) D_{L-1}(j)$

where $\mathrm{j}$ and $\mathrm{L}$ represent the translation parameter in the time domain and the inflation or deflation of time scale wavelet function, respectively. As represent in Eq.1 and Eq.2, $\mathbf{g}_{\mathbf{o}}$ is related to the scaling function $\theta$ (often called father wavelet function) and $\mathbf{h}_{\mathbf{o}}$ is related to the mother wavelet function $\psi$. Both functions can be defined as

$\theta_{L, j}(n)=2^{-\frac{L}{2}} \theta\left(2^{-j} n-j\right)$
$(3)$
$\psi_{L, j}(n)=2^{-\frac{L}{2}} \psi\left(2^{-j} n-j\right)$

(4)

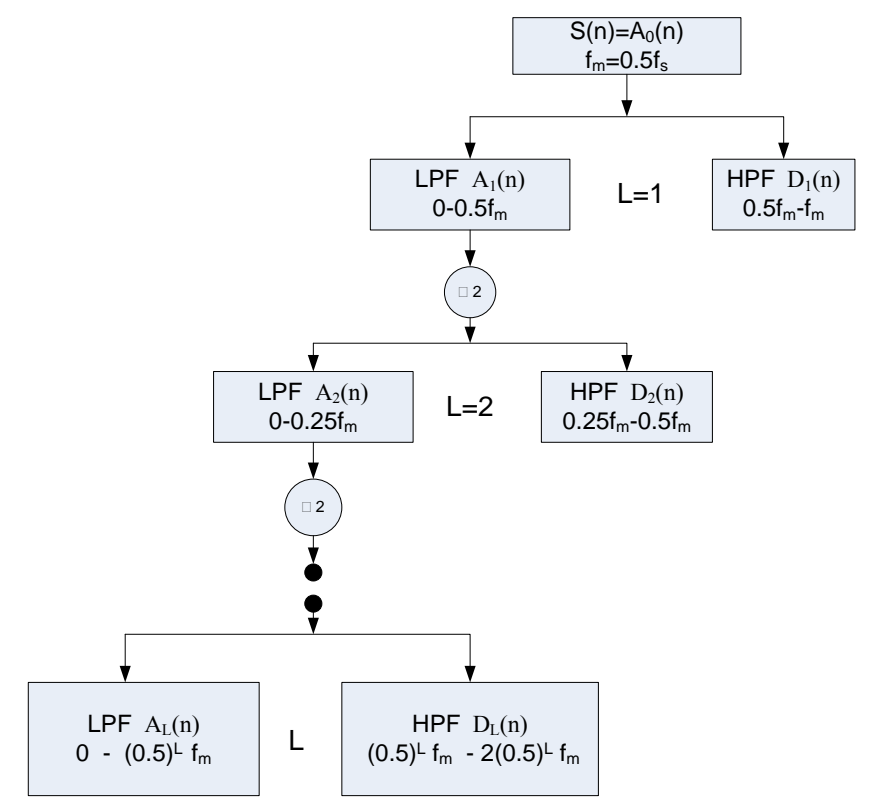

Fig. 2 The L level of repeating DWT decomposition of signal S(n). At each $\mathrm{L}$, the signal is decomposed into approximations $\left(\mathrm{A}_{\mathrm{L}}\right)$ and detail $\left(D_{L}\right)$, decimated by $2(\downarrow 2)$ relative to $A_{L-1}$. $f_{s}$ is sampling frequency

At $\mathrm{L}=1, \mathrm{~A}_{0}(\mathrm{n})$ is processed through an LPF and HPF to obtain coefficient subset $A_{1}(n)$ and $D_{1}(n)$, that are decimated by $2(\downarrow 2)$ relative to $A_{0}(n)$. The approximation coefficients are then processed to the second stage to repeat the procedure. Finally, the signal is decomposed at a targeted level (L). The illustration of decomposition tree of DWT is shown in Fig. 2.

The general process for optimal denoising of $S(n)$ needs the selection of four parameters of processing steps: 
1) Determining the type of wavelet basis function and wavelet decomposition level L.

2) Selecting the coefficient of DWT for signal reconstruction. The DWT coefficients at $\mathrm{L}$ have the specific frequency range of signal of $0-0.5^{\mathrm{L}} \mathrm{f}_{\mathrm{m}}$ for $\mathrm{A}_{\mathrm{L}}(\mathrm{n})$ and $0.5^{\mathrm{L}} \mathrm{f}_{\mathrm{m}}-2\left(0.5^{\mathrm{L}} \mathrm{f}_{\mathrm{m}}\right)$ for $\mathrm{D}_{\mathrm{L}}(\mathrm{n})$. Removing a frequency range of $\mathrm{S}(\mathrm{n})$ due to motion artefact is considered to select DWT coefficients $\left\{\mathrm{A}_{\mathrm{L}}, \mathrm{D}_{\mathrm{L}-1}, \mathrm{D}_{\mathrm{L}-2}, \ldots, \mathrm{D}_{1}\right\}$.

3) Determining the threshold function, selection rule of threshold and the threshold rescaling method of selected set of DWT coefficients.

4) The final step of signal reconstruction (denote by $S_{F}(n)$ ) is targeted to improve SNR of signal transition between relaxation to contraction and vice versa.

\section{Method of EMG signal segmentation}

The general steps of the proposed EMG segmentation is presented in Fig. 3. In this work, $\mathrm{S}(\mathrm{n})$ is acquired using the lowest tolerable limit of sampling frequency $\left(\mathrm{f}_{\mathrm{s}}\right)$ for EMG in about $256 \mathrm{~Hz}$ [19]. Based on various report studies on DWT for EMG signal, the wavelet basis function based on Daubechies's (dB45) is chosen. In step 1 (see Fig. 3), denoising of $\mathrm{S}(\mathrm{n})$ starts with removing noise due to motion artefact, with DWT decomposition with $\mathrm{L}=5$ and selected DWT coefficients. Before reconstruction, these wavelet coefficients are selected using the 'sqtwolog' function, soft threshold and rescaling using a noise estimation based on the first-level coefficients.

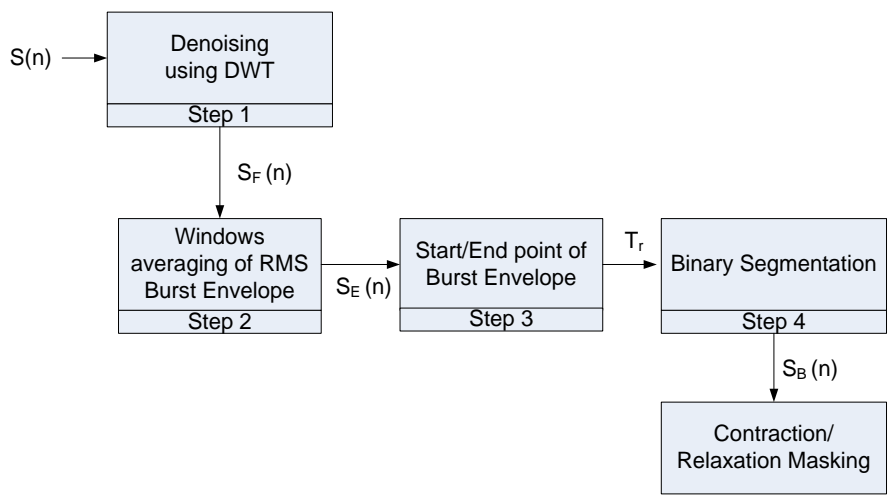

Fig. 3 Summaries of processing steps for automatic segmentation

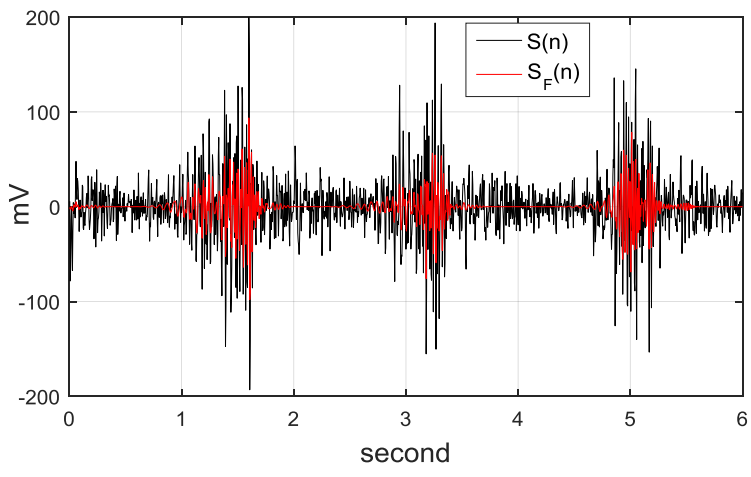

Fig. 4 EMG (S(n)) and denosing EMG $\left(\mathrm{S}_{\mathrm{F}}(\mathrm{n})\right)$
The example of signal reconstruction of thresholding DWT coefficients $\left\{\mathrm{D}_{4}, \mathrm{D}_{3}, \mathrm{D}_{2}\right.$ and $\left.\mathrm{D}_{1}\right\}$ is shown in Fig. 4. Compared with $\mathrm{S}_{\mathrm{n}}(\mathrm{f})$, resulting denoising EMG $\mathrm{S}_{\mathrm{F}}(\mathrm{n})$ could reduce the background noise in between cyclic burst as well as preserving the shape of burst signal.

In step 2, the root mean square (RMS) of $\mathrm{S}_{\mathrm{F}}(\mathrm{n})$ is used to reverse the polarity of the negative peaks into the positive peaks, without losing the information of a time interval of EMG burst. The RMS of $S_{F}(n)$ is determined by:

$S_{R}(n)=\sqrt{S_{F}(n)^{2}}$

The enveloping process starts with setting the sliding window of length $w$ samples in the $S_{R}(n)$. The envelope signal at the data points $n$ is determined by:

$S_{E}(n)=\frac{1}{N} \sum_{i=n-\frac{w}{2}}^{n+\frac{w}{2}} S_{R}(i) \cdot h(n-i)$

where $\mathrm{h}($.$) is the rectangular window, \mathrm{N}$ is number of point data in window length.
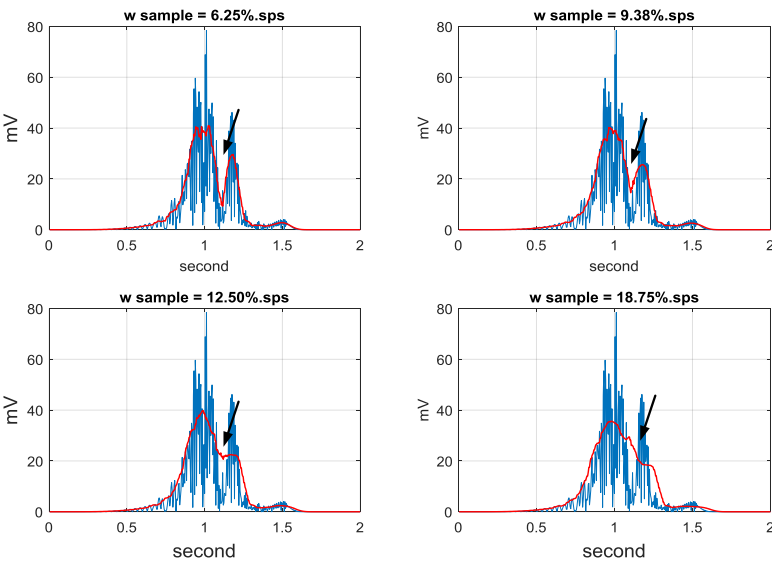

Fig. 5 Plot of $S_{R}(n)$ with superimposed enveloping RMS $S_{E}(n)$. The envelope extraction over all trend of the burst signal used sliding window with $w$ sample $12.5 \%, 9.38 \%, 12.5 \%$ and $18.75 \%$ sps. Black arrow marked a small fluctuations that may arise in a period of muscle contraction

Selection the length of $w$ must consider two aspects of the prediction of the shape of envelope $S_{R}(n)$, i.e.

1) Consistency of determination of the start/end point of $\mathrm{S}_{\mathrm{E}}(\mathrm{n})$ under small fluctuation of background noise.

2) The envelope shape of $S_{E}(n)$ must accommodate a small fluctuation that may arise in a period of muscle contraction. These fluctuations may occur due to unstable firing rates of the motor units and motion artifacts that are not optimally removed in wavelet denoising.

As an illustration, the shape of envelope $\mathrm{S}_{\mathrm{E}}(\mathrm{n})$ on burst $\mathrm{S}_{\mathrm{R}}(\mathrm{n})$ in one muscle contraction period is shown in Fig. 5. The effect of length of $w$ in $6.25 \%, 9.38 \%, 12.5 \%$ and $18.75 \%$ from sample per second (sps) are demonstrated. Note, the sps 
represents frequency sampling (fs) in Hz. With consideration of trade-off for $\mathrm{S}_{\mathrm{E}}(\mathrm{n})$ prediction, $w$ with $12.5 \%$ sps is considered for the next processing step.

For repetitive contractions, the $\mathrm{S}_{\mathrm{E}}(\mathrm{n})$ pattern produces maximum amplitude and burst duration that depends on contraction characteristic, such as the strength and the endurance of hand muscle gripping load (See Fig. 6-I). A threshold value $\left(T_{R}\right)$ must be defined to detect the location of a start/end point of a cyclic burst signal. In step 3, on the segmentation scheme, an adaptive $T_{R}$ with accommodation of a varying pattern of burst describe as follows. For common repetitive movements exercise, a number of contractions in the certain duration TD is determined on the specific scenario as follow.

1) We defined set of $N$ points data $\mathrm{S}_{\mathrm{E}}(n)$ in each TD duration, and ordered values from the least to the greatest that is represented as $\left\{\mathrm{S}_{\mathrm{Emin}-\max -\mathrm{n}}\right\}=\left\{\mathrm{S}_{\mathrm{E}}(\mathrm{n}), \ldots \ldots \mathrm{S}_{\mathrm{E}}(\mathrm{n}+\mathrm{TD})\right\}$. The plot example of $\left\{\mathrm{S}_{\mathrm{Emin}-\mathrm{max}-\mathrm{n}}\right\}$ on three different $\mathrm{TD}\left(\mathrm{TD}_{1}\right.$, $\mathrm{TD}_{2}, \mathrm{TD}_{3}$ ) is shown in Fig. 6-II. On these plots, a turning point of value from the least to the greatest may be indicated as a $T_{r}$ candidate.
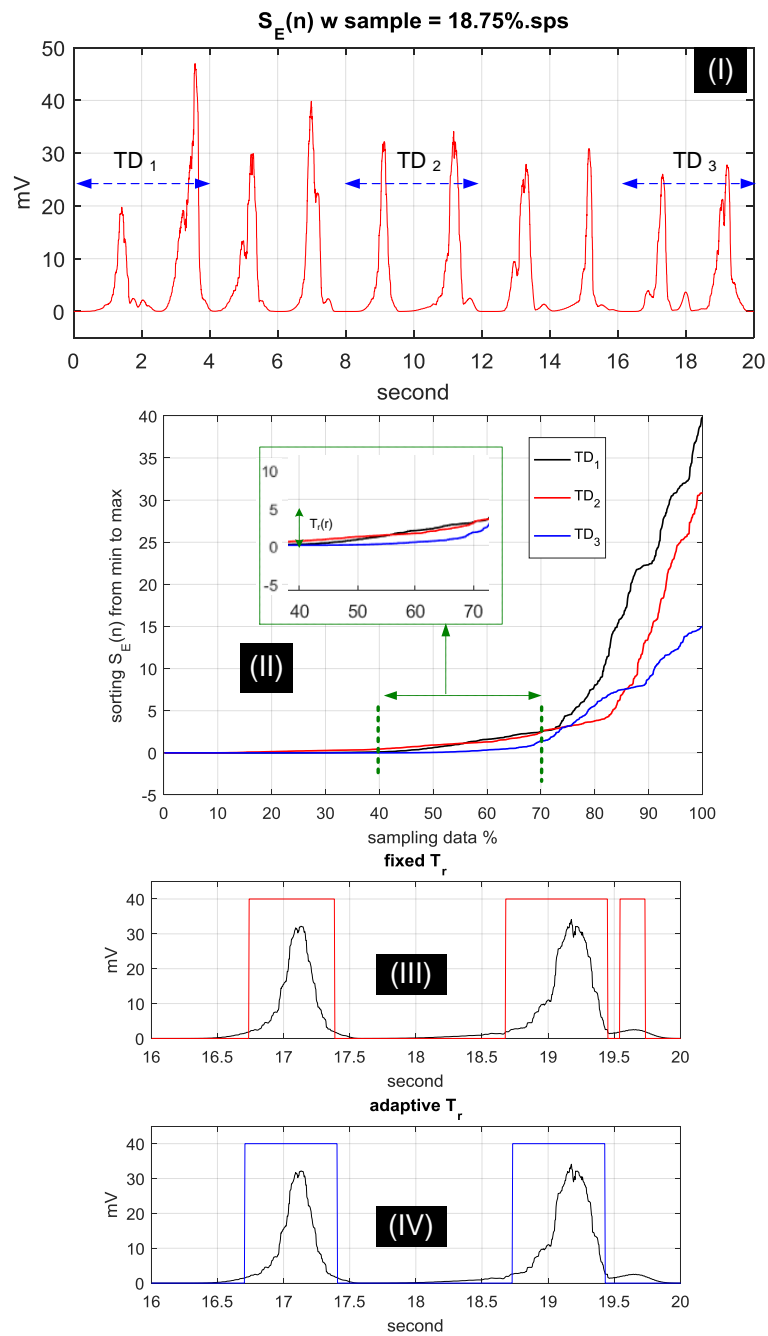

Fig. 6 The examples of a pattern of $\mathrm{S}_{\mathrm{E}}(\mathrm{n})$ in repetitive contraction (I) set of $\left\{S_{\text {Emin-max-n }}\right\}$ in a specific TD1,TD2 and TD3 (II), determined binary segmentation $\mathrm{S}_{\mathrm{B}}(\mathrm{n})$ using fixed $\operatorname{Tr}$ (III) and determined binary segmentation $\mathrm{S}_{\mathrm{B}}(\mathrm{n})$ using adaptive $\operatorname{Tr}(\mathbf{I V})$

2) Formally, a $T_{r}$ is determined based on the nearest rank method of P-th percentile $\left\{\mathrm{S}_{\text {Emin-max-n }}\right\}$ that is calculated using $\mathrm{m}=[0.01 \mathrm{P} \times \mathrm{N}]$. Then $\mathrm{T}_{\mathrm{r}}$ is determined from ordinal rank $m$ of $\left\{\mathrm{S}_{\text {Emin-max-n }}\right\}$ [21].

3) The value of $P$ can be defined based on characteristic of $\left\{\mathrm{S}_{\mathrm{Emin}-\mathrm{max}-\mathrm{n}}\right\}$ in $\mathrm{P}$ with range of $50-60$, for determined $\mathrm{T}_{\mathrm{r}}(\mathrm{n})$ in each TD.

Given an adaptive value of $T_{\mathrm{r}}$, in step 4 (see Fig. 4), a binary segmentation $\left(\mathrm{S}_{\mathrm{B}}(\mathrm{n})\right)$ of $\mathrm{S}_{\mathrm{E}}(\mathrm{n})$ is determined based on:

$S_{B}(n)=1$ if $S_{E}(n) \geq T_{r}(n)$

$S_{B}(n)=0$ if $S_{E}(n)<T_{r}(n)$

As an illustration, given $\mathrm{T}_{\mathrm{r}}(\mathrm{n})$ from $\mathrm{P}=50$ from percentile $\left\{S_{\text {Emin-max-n }}\right\}$ in TD1, a false of $\mathrm{S}_{\mathrm{B}}(\mathrm{n})$ on $\mathrm{TD}_{2}$ is demonstrated (See Fig. 6-III). In this case, the endpoint of contraction is missed to be detected. Using a value of $\mathrm{T}_{\mathrm{r}}(\mathrm{n})$ based on specific distribution $\left\{\mathrm{S}_{\mathrm{Emin}-\mathrm{max}-\mathrm{n}}\right\}$ on each $\mathrm{TD}$, it could solve false problem on $\mathrm{S}_{\mathrm{B}}(\mathrm{n})$ (See Fig. 6-IV).

\section{EXPERIMENTS AND ANALYSIS}

\section{A. Experimental data collection}

The simultaneous EEG and EMG are acquired using biosensing device based on the ADS1299 analog front-end as bio-amplifier with 8 channels and programmable gain. The packaged data are sent to computer using a wireless Bluetooth, with maximum sampling rate in about $256 \mathrm{~Hz}$. In the experiments, the disposable surface electrodes for EMG and gold cup surface electrodes for EEG are placed on the hand and scalp of head, respectively. The biosensing device is customized to acquire simultaneous EEG and EMG from the region of flexor digitorum superficialis (FDS) of muscle and cortical motor (Fz, Cz and Pz) of the brain (See Fig. 7).

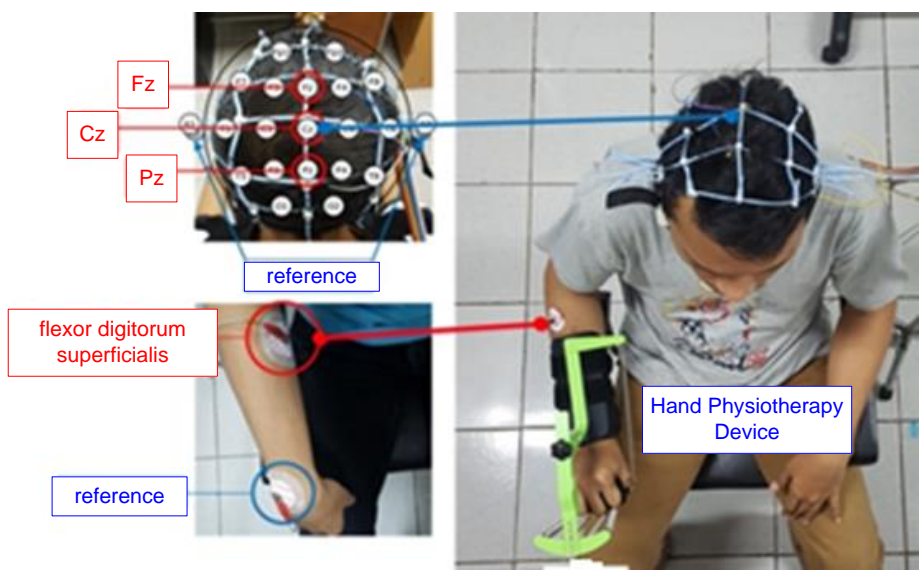

Fig. 7 Experimental data collection for simultaneous recording of EEG and EMG 
The right-handed healthy male subjects ( 22 years old) are participated in the experiment. The subject is asked to perform repetitive motor contraction tasks. With color stimulus guiding in monitor, the subject is requested to hold and release the grasp of hand physiotherapy device (See Fig. 7) twice in $\mathrm{TD}=2$ seconds duration. The activity repeats for about 5 minutes for simultaneous recording of EEG and EMG.

\section{B. Evaluation Automatic Segmentation Performance}

Performance evaluation is done by calculating the number of EMG burst that occur for the duration of repetitive movement. As an illustration, the typical EMG signal for 4TD duration (in about 8 seconds) during the repetitive exercise using hand physiotherapy device is shown in Fig. 8 .

Evaluation is done after the baseline recording stage in about 60 seconds. Then, the total number of bursts occurrences is calculated for each repetitive movement on duration each periode 40 second. Using manual counting, the total number of bursts due to contraction is 248 times. The automatic segmentation is evaluated to count and segment cyclic bursts. Based on the data set $\left\{\mathrm{S}_{\text {Emin-max-n }}\right\}$ in the initialized periode of $4 \mathrm{TD}$, fixed $\mathrm{T}_{\mathrm{r}}$ is determined using $\mathrm{P}=55$. The total errors of fail burst detection automatic segmentation with fixed threshold $\operatorname{Tr}$ is $28.5 \%$.
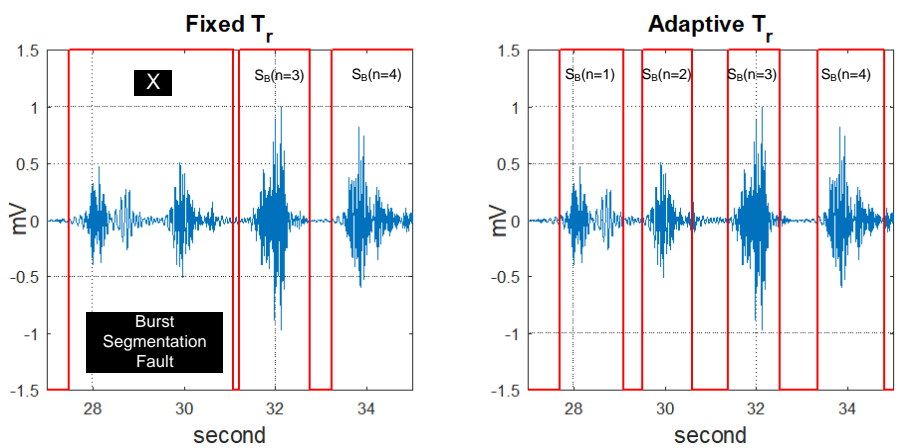

Fig. 8 The typical EMG signal for 4TD duration during the tasks which the subject is requested to hold and release grasp of hand physiotherapy device. In duration of 8 seconds, with manual segmentation, 4 burst signals can be counted. In the illustration, fixed $T_{r}$ segmentation only counts 2 contraction phase and adaptive

$\mathrm{T}_{\mathrm{r}}$ segmentation successfully to detects 4 contraction phases

Table 1. Comparison of counting EMG burst due to repetitive movement using manual, fixed $T_{r}$ segmentation and adaptive $T_{r}$ segmentation. Hold and release grasp of hand physiotherapy device is take account start from 60 second after baseline activities

\begin{tabular}{|c|c|c|c|}
\hline $\begin{array}{c}\text { Point Data } \\
\text { Sample for } \\
\text { each 40 } \\
\text { seconds }\end{array}$ & $\begin{array}{c}\text { Manual } \\
\text { (total number } \\
\text { of bursts } \\
\text { detected) }\end{array}$ & $\begin{array}{c}\text { Fixed } \mathbf{T}_{\mathbf{r}} \\
\text { (total number } \\
\text { of bursts } \\
\text { detected) }\end{array}$ & $\begin{array}{c}\text { Adaptive } \mathbf{T}_{\mathbf{r}} \\
\text { (total number } \\
\text { of bursts } \\
\text { detected) }\end{array}$ \\
\hline 1 & 20 & 17 & 18 \\
\hline 2 & 24 & 17 & 22 \\
\hline 3 & 26 & 24 & 24 \\
\hline 4 & 24 & 18 & 22 \\
\hline 5 & 24 & 19 & 18 \\
\hline 6 & 24 & 17 & 24 \\
\hline 7 & 26 & 14 & 26 \\
\hline 8 & 25 & 15 & 25 \\
\hline
\end{tabular}

\begin{tabular}{|c|c|c|c|}
\hline 9 & 27 & 22 & 26 \\
\hline 10 & 28 & 15 & 27 \\
\hline
\end{tabular}

As a comparison, with set of $\mathrm{P}=55$, adaptive threshold $\operatorname{Tr}(\mathrm{n})$ with a value on data distribution of $\left\{S_{\text {Emin-max-n }}\right\}$ on each $4 T D$ cyclic, the total errors of fail burst detection can be reduced to $6.5 \%$.

Furtherly, the results of the number of contractions determination for each sample data point and total data point, as well as the percentage of failed burst counting using manual and automatic segmentation are tabulated in Table 1.

\section{Method of EMG-EEG Quantification}

A change of strength of muscle contraction is determined based on the total RMS power of $\mathrm{S}_{\mathrm{F}}(\mathrm{n})$ on each duration $4 \mathrm{TD}$ in about 8 seconds. A power of $\mathrm{S}_{\mathrm{F}}(\mathrm{n})$ is estimated using estimation power spectral density (PSD) using periodogram Welch $[19,20]$.

Suppose, the segmented contraction of $\mathrm{S}_{\mathrm{F}}(\mathrm{n})$ are partitioned with length of $D=4 T D$. They overlaps with one another, so that one segment of the next segment is displaced along $\mathrm{S}$ data $(\mathrm{S} \leq \mathrm{D})$. Thus, the maximum $P=(N-D) / S+1$. The obtained $S_{F}^{(p)}(k)$ are:

$$
S_{F}^{(p)}(k)=w(k) S_{F}(k+p S) \cdot S_{B}(k+p S), \quad 0 \leq k \leq D-1
$$

where, $0 \leq p \leq P-1$. From those data, it is obtained:

$$
\tilde{P}_{x x}^{(p)}(f)=\left|\sum_{k=0}^{D-1} S_{F}^{(p)}(k) \exp (-j 2 \pi f s)\right|^{2}
$$

At frequency range of $-1 / 2 f s \leq f \leq 1 / 2 f s$, and $w(\mathrm{k})$ is Hamming windows. Eventually, the PSD estimation is given by:

$$
P_{W}\left(f_{i}\right)=\frac{1}{P} \sum_{p=0}^{P-1} \tilde{P}_{x x}^{(p)}(f)
$$

Average of $\mathrm{P}_{\mathrm{W}}\left(\mathrm{f}_{\mathrm{i}}\right)$ for $\mathrm{f}_{\mathrm{i}}$ is used to calculate RMS power of EMG.

Quantification analysis of EEG in relation to motoric activities is evaluated using RMS power of the brain wave for type of $m u$-wave on the frequency range of 8-13 Hz. PSD of EEG signal from channel $\mathrm{Fz}, \mathrm{Cz}$ and $\mathrm{Pz}$ is processed using PSD estimation as formulated in Eq. 9, with changed variable $\mathrm{S}_{\mathrm{F}}(\mathrm{n})$, and signal from three channels representing the motor cortex that denoted by $\mathrm{S}_{\mathrm{F}-\mathrm{FZ}}(\mathrm{n}), \mathrm{S}_{\mathrm{F}-\mathrm{CZ}}(\mathrm{n})$ and $\mathrm{S}_{\mathrm{F}-\mathrm{PZ}}(\mathrm{n})$. The power of $m u$-wave from PSD of EEG is evaluated based on the average of $\mathrm{P}_{\mathrm{W}}\left(\mathrm{f}_{\mathrm{i}}\right)$ for $\mathrm{f}_{\mathrm{i}}$ in $m u$-wave range of $8-13 \mathrm{~Hz}$ with data length of $4 \mathrm{TD}$.

\section{RESUlt AND DISCUSSION}

The usefulness of automatic segmentation for a specific study case of EMG-EMG quantification is described in this section. The proposed scheme is tested to association analysis according to the power of EMG burst and the power of $m u$ - 
wave of EEG that is recorded on the motor cortex. Information from the segmentation of the EMG signal will be used to evaluate the $m u$-wave conditions in the $\mathrm{S}_{\mathrm{F}_{-} \mathrm{FZ}}(\mathrm{n}), \mathrm{S}_{\mathrm{F}_{\mathrm{C}} \mathrm{z}}(\mathrm{n})$, and $\mathrm{S}_{\mathrm{F}-\mathrm{PZ}}(\mathrm{n})$ signals for muscle conditions in relaxation and contraction.

Quantification of the RMS power $\mathrm{S}_{\mathrm{F}}(\mathrm{n})$ under contraction conditions for each duration of 8 seconds is presented in Fig. 9. Curve fittings provide information about muscle biofeedback during holding and releasing grasp repeatedly. In the sampled data of 20-30 (in about exercise duration from $160 \mathrm{sec}$ to $240 \mathrm{sec}$ ), muscle contraction tends to be in the maximum power then gradually decreases.

With regards to the biofeedback responses from the brain, the motor activity will be associated with the changes of the power of $m u$-wave in the motor cortex region in Frontal $(\mathrm{Fz})$, Central $(\mathrm{Cz})$, and Parietal $(\mathrm{Pz})$ [7,8,9]. The results of automatic segmentation for the synchronization of EMG-EEG quantification is shown in Fig. 9. Separation of the EEG signal $\mathrm{S}_{\mathrm{F} C Z}(\mathrm{n})$ into contraction and relaxation conditions provide information on the different $m u$-wave RMS power for both conditions.
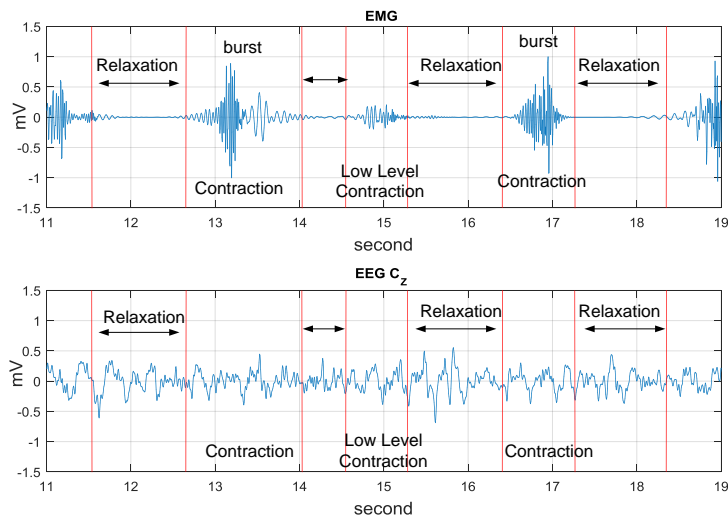

Fig. 9 Plot of example automatic segmentation for synchronization EMG-EEG

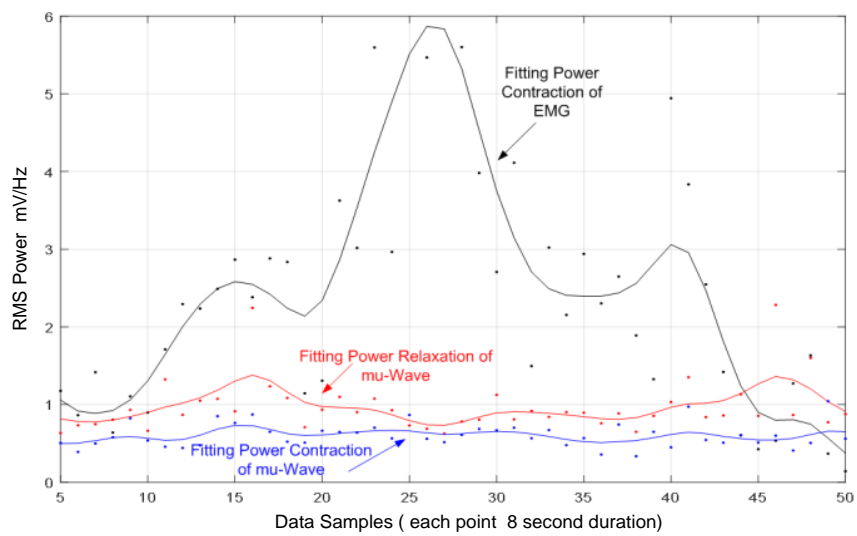

Fig. 10 Average RMS power of EMG contraction and $m u$-wave rhythm in contraction and relaxation condition

The fluctuations of the power of $m u$-wave during contraction are shown in Fig. 10, as the results of the recording on the $\mathrm{Cz}$ region. Biofeedback evaluations for the average power of muscle and brain activities in the three recording regions are shown in Fig. 10. Fig. 10 shows the average power from the measured signal activities for 40 seconds duration. In general, the power of $m u$-wave during the relaxation is higher than during the contraction conditions.

While the RMS power in the EMG tends to increase, the power of $m u$-wave between two repetitive contractions is contrarily decreasing (see Fig. 11 at the $3^{\text {rd }}$ segment of sample data). In the final segments, the brain activities show an increase in the power of $m u$-wave during relaxation (see Fig. 11 at the $3^{\text {rd }}-5^{\text {th }}$ segment of sample data). Changes in the $m u$ wave conditions during contraction have smaller dynamics $(\sigma$ $\left.= \pm 0.0637 \mathrm{mV}_{\mathrm{RMS}}\right)$, compared to relaxation conditions $(\sigma=$ $\left.\pm 0.1338 \mathrm{mV}_{\mathrm{RMS}}\right)$.
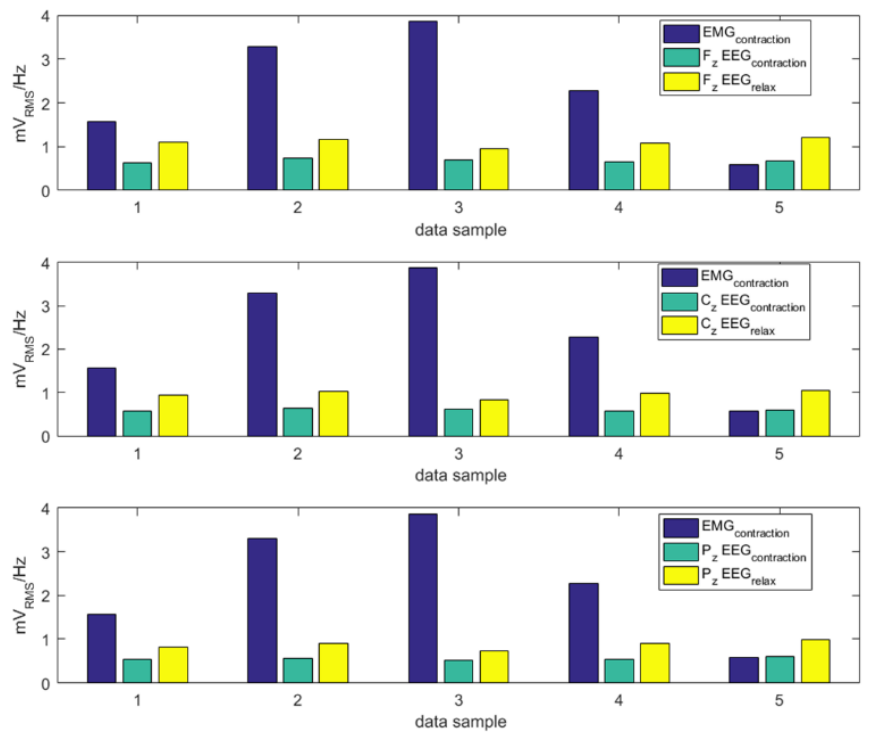

Fig. 11 Segmentation of average RMS power of EMG contraction and mu-wave rhythm on $\mathrm{Fz}, \mathrm{Cz}$, and $\mathrm{Pz}$ region

Associated with the concept of the $m u$-wave response phenomenon, under contraction conditions, the asynchronous event over the motor cortex will cause the attenuation of the power of $m u$-wave. Conversely, the synchronous condition marked by an increase in the power of $m u$-wave occurs in a state of relaxation. This condition is under the principal characteristics of the $m u$-wave, representing the relaxed state.

The study results show that the ability to separate the analysis of EEG signals in conditions of relaxation and contraction is potential as an effective for biofeedback rehabilitation exercise.

\section{CONCLUSION}

The proposed scheme utilizes the EMG signal preprocessing strategy before implementing signal enveloping based on DWT denoising methods. The DWT denoising methods are obtained on five decomposition levels, which are performed thorough consistency in determining the segment duration of related contraction. The signal enveloping process uses a sliding window with a width of $12.5 \%$ sps and an adaptive threshold. 
INTERNATIONAL JOURNAL OF BIOLOGY AND BIOMEDICAL ENGINEERING

DOI: $10.46300 / 91011.2020 .14 .19$

The proposed scheme of automatic segmentation EMG-

EEG can improve the efficiency time for biofeedback analysis, particularly for repetitive/periodic motor exercise in the medical rehabilitation devices.

In many situations, recording EMG-EEG is also influenced by the patient's condition and a typical exercise scenario of rehabilitation therapy. Therefore, determining a threshold parameter using a machine learning approach and large data sets may improve the current performance of our proposed scheme.

\section{ACKNOWLEDGMENT}

We acknowledge Institut Teknologi Bandung, Indonesia that Funding for Community Services and Innovation Research Program 2020.

\section{REFERENCES}

[1] S.M. Hatem, G. Saussez, M.D. Faille, V. Prost, X. Zhang, D. Dispa, Y. Bleyenheuft, "Rehabilitation of motor function after stroke: a multiple systematic review focused on techniques to stimulate upper extremity recovery," Frontiers in Human Neuroscience, vol. 10, pp. 442, 2016.

[2] G.D. Eisenberg, K.G.H.M. Fyvie, A-K. Mohamed, "Real-time segmentation and feature extraction of electromyography: towards control of a prosthetic hand," IFAC-PapersOnLine, vol. 50, no. 2, pp. 151-156, 2017.

[3] E.F. Shair, S.A. Ahmad, M.H. Marhaban, S.B.M. Thamrin, A.R. Abdullah, "EMG processing based measures of fatigue assessment during manual lifting," BioMed Research International, vol. 2017, 2017.

[4] N. Nazmi, M.A.B.A. Rahman, M. A., S-I. Yamamoto, S. Ahmad, H. Zamzuri, S.A. Mazlan, "A review of classification techniques of EMG signals during isotonic and isometric contractions," Sensors, Basel, 2016, vol. 16, no. 8, pp. 1304.

[5] S. Bhagwat, P. Mukherji, "Electromyogram (EMG) based fingers movement recognition using sparse filtering of wavelet packet coefficients," Sãdhanā, vol. 45, no.3, 2020.

[6] N. Yoshimura, H. Tsuda, T. Kawase, H. Kambara, Y. Koike, "Decoding finger movement in humans using synergy of EEG cortical current signals," Sci Rep, vol. 7, no. 11382, 2017.

[7] M.C.F. Castro, A.A. Masiero, F.T. Rocha and P.T. Aquino, "Motor imagery recognition and its cerebral mapping," Proc IEEE Biosignals and Biorobotics Conference, Salvador, 2014, pp. 1-5.

[8] S. Simon, R. Mukamel, "Power modulation of electroencephalogram $\mathrm{mu}$ and beta frequency depends on perceived level of observed actions," Brain and Behavior, vol. 6, no. 8, pp. e00494, 2016.

[9] R. Debnath, V.C. Salo, G.A. Buzzell, K.H. Yoo, N.A. Fox, "Mu rhythm desynchronization is specific to action execution and observation: Evidence from time-frequency and connectivity analysis," Neuroimage, vol. 184, pp. 496-507, 2019.

[10] X. Zhang, G. Hegerl, F.W. Zwiers, J. Kenyon,"Avoiding inhomogeneity in percentile-based indices of temperature extremes," J Climate, vol. 18, no. 11, pp. 1641-1651, 2005.

[11] B. Kim, L. Kim, Y-H. Kim, S.K. Yoo, "Cross-association analysis of EEG and EMG signals according to movement intention state," Cognitive Systems Research, vol. 44, pp. 1-9, 2017.

[12] E.F. Shair, A.R. Abdullah, T.N.S.T. Zawawi, S.A. Ahmad, S.M. Saleh, "Auto-segmentation analysis of EMG signal for lifting muscle contraction activities," Journal of Telecommunication,
Electronic and Computer Engineering, vol. 8, no. 14, pp. 17-22, 2016.

[13] R.H. Chowdhury, M.B.I. Reaz, M.A.B.M. Ali, A.A.A. Bakar, K. Chellappan, T.G. Chang, "Surface electromyography signal processing and classification techniques," Sensors, Basel, 2013, vol. 13, no.9, pp. 12431-66.

[14] M. Wang, X. Wang, C. Peng, S. Zhang, Z. Fan, Z. Liu, "Research on EMG segmentation algorithm and walking analysis based on signal envelope and integral electrical signal," Photonic Network Communications, vol. 37, pp. 195-203, 2019.

[15] J. Sedlak, D. Spulak, R. Cmejla, R. Bacakova, M. Chrástková, B. Kracmar, "Segmentation of surface EMG signals," Proc IEEE International Conference on Applied Electronics, Pilsen, 2013, pp. 1-4.

[16] J.A. Guerrero, M.A. Castillo-Galvan, J.E. Macías-Díaz, "Novel electromyography signal envelopes based on binary segmentation," Biomedical Signal Processing and Control, vol. 45, pp. 225-236, 2018.

[17] M. Domino, B. Pawlinski, Z. Gajewski, "Biomathematical pattern of EMG signal propagation in smooth muscle of the nonpregnant porcine uterus," PLoS One, vol. 12, no. 3, pp. e0173452, 2017.

[18] A. Merlo, I. Campanini, "Technical aspects of surface electromyography for clinicians," The Open Rehabilitation Journal, vol. 3, pp. 98-109, 2010.

[19] C. Larivière, A. Delisle, A. Plamondon, "The effect of sampling frequency on EMG measures of occupational mechanical exposure," J Electromyogr Kinesiol, vol. 15, no. 2, pp. 200-209, 2005.

[20] J. Wu, X. Li, W. Liu, Z.J. Wang, "sEMG signal processing methods: a review," Journal of Physics: Conference Series, vol. 1237, no. 3, pp. 1-6, 2019.

[21] J. Zhang, B. Wang, C. Zhang, Y. Xiao, M.Y. Wang, "An EEG/EMG/EOG-based multimodal human-machine interface to real-time control of a soft robot hand," Frontiers in Neurorobotics, vol. 13, no.7, pp. 1-13, 2019.

[22] G. Wang, Y. Zhang, J. Wang, "The analysis of surface EMG signals with the wavelet-based correlation dimension method," Comput Math Methods Med, vol. 2014, pp. 1-9, 2014.

[23] S-H. Liu, C-B. Lin, Y. Chen, W. Chen, T-S. Huang, C-Y. Hsu, "An EMG patch for the real-time monitoring of muscle-fatigue conditions during exercise," Sensors, Basel, 2019, vol. 19, no. 14, pp. 3108.

[24] Martin Strmiska, Zuzana Koudelkova, Martina Zabcikova, "Measuring Brain Signals Using Emotiv Devices", WSEAS Transactions on Systems and Control, pp. 537-542, Volume 13, 2018.

Suprijanto is Associate Professor in Instrumentation and Control Research Group, Faculty of Industrial Technology, Institut Teknologi Bandung, Indonesia (e-mail: supri@tf.itb.ac.id).

Azizah S. Noor is Graduated Master Program of Instrumentation and Control, Faculty of Industrial Technology, Institut Teknologi Bandung, (e-mail: azizahsyifalianti@gmail.com).

Ayu G. Risangtuni is Lecturer in Instrumentation and Control Research Group, Faculty of Industrial Technology, Institut Teknologi Bandung, Indonesia (e-mail: ayugareta@gmail.com).

Hesty Susanti is Researcher in Medical Instrumentation Laboratory, Instrumentation and Control Research Group, Faculty of Industrial Technology, Institut Teknologi Bandung, Indonesia (e-mail: hesty.billiton@gmail.com).

\section{Creative Commons Attribution License 4.0 (Attribution 4.0 International, CC BY 4.0)}

This article is published under the terms of the Creative Commons Attribution License 4.0 https://creativecommons.org/licenses/by/4.0/deed.en_US 